\title{
Paraganglioma of the Cauda Equina Region: A Case Report
}

\author{
Hyeongyu Jang ${ }^{1}$, Moo Sung Kang ${ }^{1}$, Sang Yeop $\mathrm{Yi}^{2}$, Kwang Ryeol Kim¹, Byung Ho Jin ${ }^{1}$ \\ Departments of ${ }^{1}$ Neurosurgery, ${ }^{2}$ Pathology, International St. Mary's Hospital, Catholic Kwandong University Collage of \\ Medicine, Incheon, Republic of Korea
}

Corresponding author:

Byung Ho Jin

Department of Neurosurgery,

International St. Mary's Hospital,

Catholic Kwandong University

Collage of Medicine, 25,

Simgok-ro 100beon-gil, Seo-gu,

Incheon 22711, Republic of Korea

Tel: +82-32-290-3079

Fax: +82-32-290-3879

E-mail: bhjinccf@hanmail.net

Received: April 29, 2020

Revised: June 18, 2020

Accepted: June 19, 2020
Paraganglioma is a rare neuroendocrine tumor that is assumed to be derived from paraganglia. This report describes the clinical presentation and treatment outcome of a paraganglioma in the cauda equina region. A 47-year-old female patient presented with 2 months of lower back pain and severe radiating pain in the extremities of both legs. Magnetic resonance imaging of the lumbosacral spine disclosed an oval-shaped lesion measuring $2.6 \times 1.4 \times 1.5 \mathrm{~cm}$ at the $\mathrm{L} 3$ level. T2-weighted images (WIs) revealed a high signal intensity lesion with signal void capping the tumor, and T1-WI demonstrated a slightly low signal. Gross total removal of the tumor was performed without any intraoperative hyperadrenergic signs. Pathological findings showed Zellballen appearance and immunochemistry with antisera to synaptophysin showed that the tumor was consistent with neuroendocrine lineages, indicating a chromaffin cell origin. Clinically, the patient showed improvement of lower back and radiating pain, without any neurological deficit postoperatively. This case emphasizes the importance of preoperative suspicion of paraganglioma, as surgery is the safest way to remove the lesion. Excellent results can be obtained with surgical removal in most cases of paraganglioma.

Key Words: Cauda equina; Lumbar vertebrae; Neoplasms; Paraganglioma

\section{INTRODUCTION}

The term paraganglioma usually refers to rare neuroendocrine tumors that originate in extra-adrenal autonomic paraganglia. The first description of paraganglioma in the cauda equina region of paraganglioma was provided by Lerman et al. ${ }^{7)}$ in 1972, and this pathologic entity is now known to account for $3.5 \%$ to $4 \%$ of all cauda equina tumors. The incidence in the general population is thought to be 0.07 per 100,000 population ${ }^{1,6,7)}$.

In this report, we discuss a case of a middle-aged woman with axial and radicular pain secondary to a spinal cord tumor, which was revealed by surgery to be a paraganglioma.

\section{CASE REPORT}

A 46-year-old woman presented with a 2-month history of lower back pain and radiating pain in both lower extremities, with a visual analog scale score of 10 . Radiating pain was confined to both the buttock and posterior thigh, which corresponded to the S1 dermatome. For neurological evaluation, she was graded with an American Spinal Injury Association Impairment Scale score of E, and sphincter dysfunction was absent. She was referred to our clinic with suspicion of an intradural extramedullary spinal cord tumor. Magnetic reso- nance imaging (MRI) of the lumbosacral spine disclosed an oval-shaped lesion measuring $2.6 \times 1.4 \times 1.5 \mathrm{~cm}$ at the $\mathrm{L} 3$ level. T2-weighted images (WIs) revealed a high signal intensity lesion with a signal void capping the tumor, and T1-WI demonstrated a slightly low signal. The tumor showed homogeneous enhancement after gadolinium injection (Fig. 1).

The patient underwent an L3 laminotomy, and after midline standard durotomy, the tumor nodule was exposed, deep within the cauda equina roots. The cranial and caudal tumor-
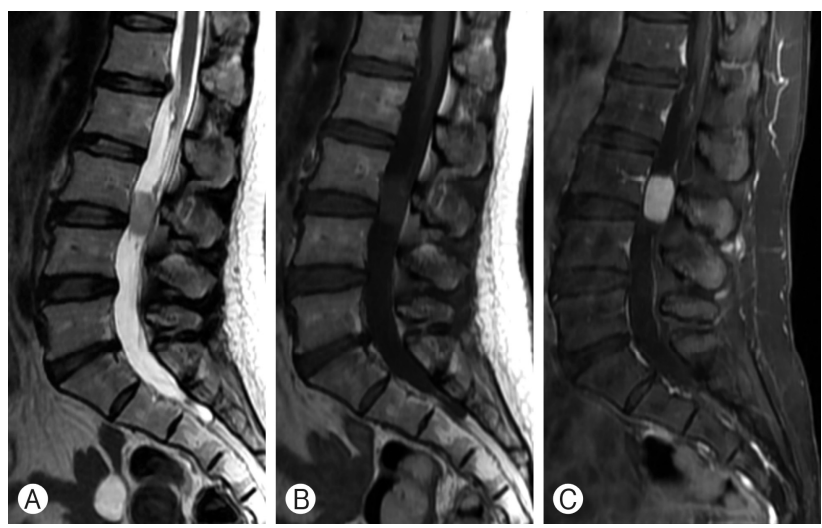

Fig. 1. Preoperative magnetic resonance imaging of the lumbar spine, sagittal views: sagittal T2-weighted image $(\mathrm{WI})(\mathrm{A})$, T1-WI (B), T1-WI with contrast $(\mathrm{C})$. 

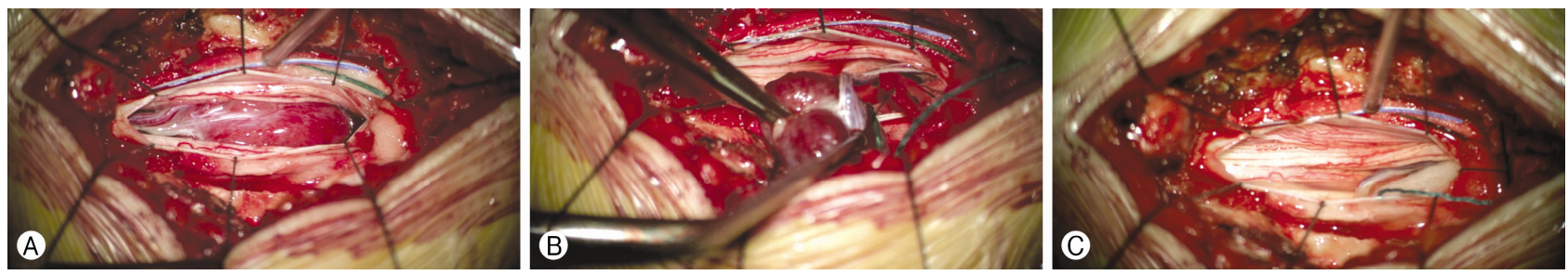

Fig. 2. Intraoperative images showing reddish oval-shaped tumor with cauda equina and vascular pedicle attached to the cranial head of tumor (A). Although the tumor was hypervascularized, it was well encapsulated and could be removed en bloc (B). There was no residual bleeding after tumor removal. The truncated cauda equina is shown (C).
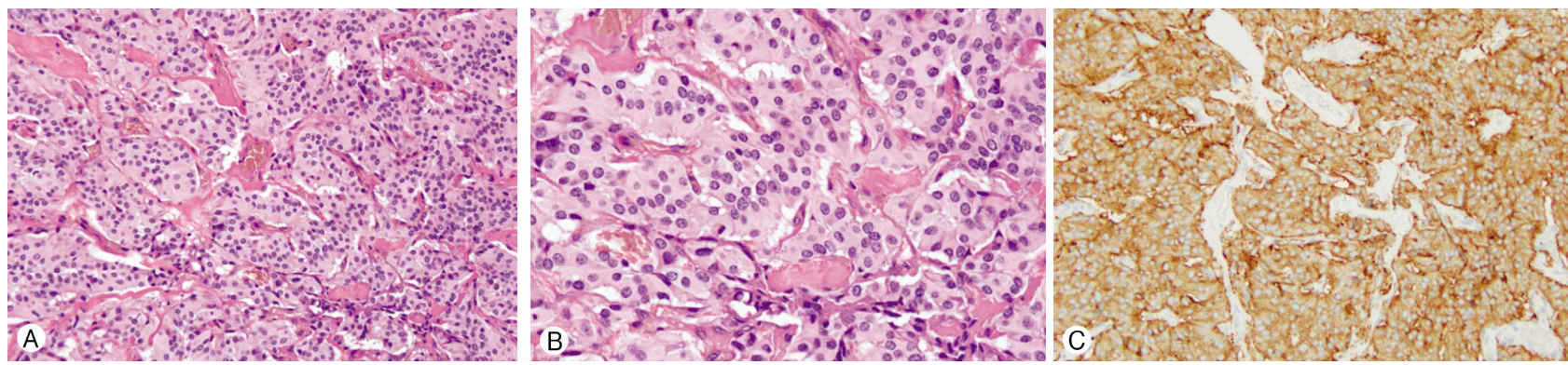

Fig. 3. (A) Photomicrograph showing the nest of tumor cells consisting of round small tumor cells, separated by a fine vascular network, known as the Zellballen pattern (hematoxylin and eosin stain [H\&E], $\times 200$ magnification). (B) The reticulin staining highlighted this pattern; tumor cells showed a pseudorosette pattern, and the nuclei were uniformly round to oval (H\&E, $\times 400$ magnification). (C) Immunohistochemical staining was positive for synaptophysin.

adhering cauda equina and vessel were resected, and the tumor was removed en bloc.

The tumor appeared to be well encapsulated and hypervascularized (Fig. 2). During the surgical procedure, systemic hyperadrenergic signs were not observed. The axial and radiating pain improved immediately. Gadolinium-enhanced postoperative MRI of the lumbosacral spine verified the total excision of the lesion.

On microscopic inspection, the sections showed a Zellballen pattern, which is a loose cluster of cells separated by a fine vascular stroma. Histopathological diagnosis was based on immunohistochemical staining with a positive reaction for synaptophysin (Fig. 3).

The patient improved immediately after surgical treatment and was discharged on postoperative day 14. At the 6-month follow-up, the patient had no clinical signs of radiculopathy.

\section{DISCUSSION}

Paragangliomas are rare neuroendocrine tumors of neuroepithelial origin that are derived from the neural crest. The paraganglia of the embryonic sympathetic and parasympathetic nervous system are composed of neuroendocrine cells called chromaffin cells. At 12 weeks of gestation, differentiation and migration of chromaffin cells is complete, and both the adrenal medulla and paraganglia are well developed. After birth, the paraganglia gradually disappear by 3 years of age. Having an identical differentiation process, pheochromocytoma is closely histologically related to paraganglioma.

Paraganglia have either parasympathetic or sympathetic function. A sympathetic ganglion consists of chromaffin cells that can synthesize and secrete catecholamines, including norepinephrine. Sympathetic paraganglia are usually located in the abdomen (75\%), bladder and prostate (10\%), and skull base (5\%). However, parasympathetic paraganglia are usually nonfunctional and are mainly distributed around the jugular and nodose ganglion. Paragangliomas arising outside of the skull base and neck are mostly sympathetic and secrete catecholamine, resulting in hyperadrenergic symptoms, including hypertension, episodic headache, sweating, and tachycardia. One study reported a ratio of norepinephrine hypersecretion of up to $86 \% \%^{4)}$. However, paragangliomas in the cauda equina rarely show hyperadrenergic systemic symptoms, which are common in pheochromocytoma. Lack of hyperadrenergic symptoms is assumed to result from the inability of tumor cells to release catecholamines into the bloodstream, or the inability of chemicals to induce symptoms ${ }^{5}$. Corinaldesi et al. ${ }^{2)}$ analyzed 234 cases of paraganglioma in the cauda equina and reported that the most common presentation was lower back pain, with or without sciatica, and clinical manifestations were similar to other types of tumors that occur in the same region. Cauda equina syndrome was seen in only $2.9 \%$ of cases. The incidence showed male dominance (male : female ratio was $1.4: 1$ ), and patients ranged from 9 to 77 years of age, peaking in the 4 th or 5 th decade ${ }^{2)}$.

The diagnosis of a paraganglioma usually starts with measure- 
ments of urinary and/or plasma fractionated metanephrines and catecholamines, although these tools are not effective for paraganglioma in the cauda equina. Radiologic imaging, especially MRI, is the gold standard of assessment. Paragangliomas are hypervascular lesions and may have flow voids around a matrix of increased signal intensity. Intralesional punctate or serpiginous signal voids on the edge of the tumor represent dilated feeding vessels. The tumor itself seems iso- or hypointense on T1-WIs and hyperintense on T2-WIs. Contrast-enhanced T1-weighted sequences demonstrate marked enhancement. Paramagnetic effects from a hemorrhagic appendage may darken the margin of the tumor. However, precise preoperative diagnosis is still problematic because of the absence of pathognomonic findings. Myxopapillary ependymomas, ependymomas, schwannomas, and meningiomas should be considered as differential diagnoses.

Grossly, the tumor is a reddish and well-circumscribed mass with adherence to the nerve root or conus medullaris or vascular pedicle $^{8)}$. Histopathological examination uniformly demonstrates nests of small, polyhedral, round, or cuboidal cells, known as the Zellballen pattern, which is separated by a dense connective tissue and vascular network. The predominant cell type is the chief cell with ample eosinophilic granular cytoplasm, and mitosis is rarely observed ${ }^{1)}$. Using light microscopy, it is not easy to distinguish between paragangliomas and ependymomas, which have a typical radial perivascular arrangement of cells called pseudorosettes. Immunohistochemical stains, including the use of several antisera, complete the diagnostic algorithm. Neuronal or neuroendocrine tumor markers, including synaptophysin or neuron-specific enolase, are usually strongly positive. The reaction with chromogranin, a pro-hormone related to neuroendocrine function, is also highly positive. Staining with epithelial membrane antigen, which stains regions of mesenchymal or epithelial origin, is rarely positive. The S-100 protein with neural crest origin has a diffusely positive reaction in supportive tissue, and staining with glial fibrillary acid protein is also negative.

According to the case review, resection is preferred as the first treatment modality for a paraganglioma in the cauda equina. A presumptive preoperative diagnosis of a paraganglioma based on clinical, biochemical, and radiographic results is pivotal in surgical planning. Before resection, the likelihood of catecholamine secretion should be considered even in cases without hyperadrenergic symptoms. However, intraoperative systemic hyperadrenergic manifestations of paragangliomas in the cauda equina have rarely been reported (4 out of 234 cases) and these symptoms subsided when the vascular pedicle was clamped ${ }^{2)}$.

Gross total removal (GTR) was achieved in more than 80\% of cases, and subtotal resection (STR) was performed in less than $10 \%$ of cases $^{2}$. The overall prognosis was excellent after GTR, and recurrence was infrequent irrespective of additional radiotherapy. However, local recurrence after STR was observed frequently, especially in patients without adjuvant radio- therapy. In most cases with recurrence or regrowth of tumors, further surgery was performed with the second round of radiotherapy. Only $1.7 \%$ of metastases from paraganglioma of the cauda equina have been reported.

Histological features such as nuclear pleomorphism, necrosis, mitotic rate, and local invasion are not useful for predicting unfavorable outcomes. According to the 2004 World Health Organization criteria, metastatic spread itself is the only true indicator of malignant behavior ${ }^{3)}$.

\section{CONCLUSION}

paraganglioma in the cauda equina region is a rare, low-grade neuroendocrine neoplasm that arises from paraganglionic tissue. It usually produces sciatica through the effect of its mass on the cauda equina. It is not easy to distinguish between paraganglioma and other intradural extramedullary tumors using radiographical studies. Preoperative suspicion of paraganglioma cannot be emphasized enough because it can infrequently cause hyperadrenergic symptoms during surgery.

\section{CONFLICTS OF INTEREST}

No potential conflict of interest relevant to this article was reported.

\section{REFERENCES}

1. Aghakhani N, George B, Parker F: Paraganglioma of the cauda equina region-report of two cases and review of the literature. Acta Neurochir (Wien) 141:81-87, 1999

2. Corinaldesi R, Novegno F, Giovenali P, Lunardi T, Floris R, Lunardi P: Paraganglioma of the cauda equina region. Spine J 15:e1-e8, 2015

3. DeLellis RA, Lloyd RV, Heitz PU, Eng C: Pathology and genetics of tumours of endocrine organs. World Health Organization Classification of Tumours. Lyon, FR: IARC Press, 2004

4. Erickson D, Kudva YC, Ebersold MJ, Thompson GB, Grant CS, van Heerden JA, et al.: Benign paragangliomas: clinical presentation and treatment outcomes in 236 patients. J Clin Endocrinol Metab 86:5210-5216, 2001

5. Gould VE, Valaitis J, Trujillo Y, Chejfec G, Gruhn JG: Neuroendocrinoma of the jejunum: Electron microscopic and biochemical analysis. Cancer 46:713-717, 1980

6. Landi A, Tarantino R, Marotta N, Rocco P, Antonelli M, Salvati $\mathrm{M}$, et al.: Paraganglioma of the filum terminale: Case report. World J Surg Oncol 7:95, 2009

7. Lerman RI, Kaplan ES, Daman L: Ganglioneuroma-paraganglioma of the intradural filum terminale. Case report. J Neurosurg 36:652-658, 1972

8. Lipper S, Decker RE: Paraganglioma of the cauda equina. A histologic, Immunohistochemical, and ultrastructural study and review of the literature. Surg Neurol 22:415-420, 1984 